\title{
Wireless Sensors Network for Traffic surveillance and management in Smart Cities
}

\author{
Anass El Mrini ${ }^{1 *}$ and Abdellatif Ghacham Amrani ${ }^{1}$ \\ ${ }^{1}$ Dept of Physics, Laboratory of Science and Advanced Technology, Multidisciplinary Faculty of Larache, Morocco
}

\begin{abstract}
The efficiency of a city's own transportation system heavily influences its economic growth due to the increasing need to transport labour force, consumers and cargo. The use of the latest technologies to solve traffic problems is the main theme of ITS "Intelligent transportation system", several solutions are already in use in many cities around the world and it is an indispensable part of the smart cities project. Our research focuses on developing a solution to manage road traffic in a smart city environment by utilizing wireless sensor network as medium to surveille the traffic. In this paper, we propose a set of countermeasures to avoid congestion and improve the flow of the traffic. The proposed countermeasures were simulated, and the results showed that our plan reduced the traffic congestion, minimized gases emission and increased fuel usage efficiency.
\end{abstract}

\section{Introduction}

There is a long-term dynamic interaction between transport and the nature of the city economy. Cities have economic cores explained by various forms of agglomeration economies, which are often based on a traditional industrial or trading base [1]. Cities are changing with the movement of people and businesses out from the centre, increased suburbanization, and the desire for lower residential and job densities. Suburbanization of employment has followed, and the simple local journeyto-work pattern or the public transport-based movement to the strong central area employment location has been replaced by more complex longer distance car-based movements. Traffic problems occur when the city own transport system can't cope with its transportation demands, and the bigger the city, the more it is vulnerable to traffic problems.

ITS, Intelligent Transportation Systems is a set of solutions and tools to improve transportation efficiency and safety, it also aims at improving energy saving and reducing vehicles emissions [2]. Generally, ITS can be classified into several subsystems according to their functions: Travel Management and Traveller Information System (TMTIS), Advanced Vehicle Control and Safety System (AVCSS) and Commercial Vehicle Operation (CVO). TMTIS is responsible for providing real-time traffic information, and for managing and controlling traffic condition and traffic lights.

Several WSN-based traffic surveillance systems have been proposed, Cheung et al. [3] built a traffic surveillance system based on wireless sensors. The proposed system is deployed along roadsides and at intersections, it uses magnetic sensors to detect vehicle detection through disturbance in the earth's magnetic field. The system can collect traffic data such as vehicle count, occupancy, speed, vehicle classification and reidentification.

O. Karpis et al. [4] tested the applicability of WSN in traffic monitoring, two magnetic sensors nodes were placed on the verge of the road with spacing of $2 \mathrm{~m}$. Traffic parameters were accurately measured including the number and the speed of vehicles, initial experiments about estimating the length of the vehicles were also conducted, showing the possibility of the classification of vehicles.

Chen et al. [5] propose a system of WSN for Intelligent Transportation System (WITS), used for the information gathering and data transferring. In this system, each vehicle is equipped with a wireless sensor unit that measures the vehicle parameters, the roadside unit collect the data from the passing vehicles units and transfers it to the intersection unit. The roadside unit gathers the information of the vehicles around and transfers it to the intersection unit. The intersection unit analyses the received information, and forward them to the strategy sub-system, which in turn calculates an appropriate scheme according to the pre-set optimization target (such as maximum throughput, minimum waiting time, etc.). The drawback of this approach is the necessity of equipping every vehicle with a sensor node, a requirement that is very unlikely to be met in a real-life application.

M. Franceshinis et al. [6] proposes a solution, within the SAFESPOT Project, using WSN to obtain traffic related data in order to generate automatic safety warnings along the road network. The architecture follows a Master-Salve paradigm in a linear topology, the system consists of several sensor nodes that are connected to a single gateway node GN. The sensor nodes are installed 
on the roadside and are responsible for estimating vehicle count, speed and direction. the gateway node forwards the data to the road side unit RSU via a wired connection. The linear topology causes delay and scalability problems, also the wired connection between the GN and RSU becomes costly and inefficient in a large-scale application (i.e. smart city).

J.J. Fernandez et al. [7] proposes a platform called Urban Information System based on WSN in order to characterize urban traffic parameters. The platform collects traffic data by taking advantage of Bluetooth devices in the vehicles. Furthermore, it can collect environmental information such as noise levels, light intensity, pressure, temperature, humidity, airborne dust and gases. Even though the system can generate OriginDestination matrix in real-time with acceptable accuracy, the system assumes all vehicles to have a functioning Bluetooth device.

The literature shows that applying the WSN technologies in the field of intelligent transportation systems, covers the need for real-time and accurate traffic parameters. A network of wireless sensors offers flexibility, scalability, and lower installation and maintenance costs than the traditional surveillance systems. However, these studies mainly focus only on effective traffic information acquisition, but do not propose a whole system capable of traffic monitoring and management at the same time.

The remainder of this paper is organized as follows, Section II exposes the motivation for using WSN to monitor urban traffic, section III defines the functionalities of the proposed traffic management system, in section IV the results of a basic simulation of the proposed system is evaluated. Section V concludes the paper and outlines our future work.

\section{APPLICATION OF WSN IN TRAFFIC SURVEILLANCE}

ITS rely on accurate, reliable real-time traffic data. And a successful application of ITS technologies in a smart city means a large-scale deployment of surveillance systems on all major highways and local streets. Surveillance systems nowadays rely heavily on intrusive sensors inductive loops specifically, which has many undesirable features. That's why the biggest motivation for using WSN for traffic surveillance is the advantages that it presents in comparison to the other sensors technologies: - Cost: a cost comparison between different surveillance technologies - including device cost, installation and maintenance cost - shows that the cost of these networks is about half that of inductive loops [8], and it is expected to rapidly decline with volume.

- High accuracy: wireless sensors proved to be the most accurate sensor besides the inductive loops when it comes to vehicles count or speed measurement [8]. Also, by using the collaborative approach in a WSN the detection accuracy and reliability can be improved greatly. Other test also shows that vehicle classification is also possible with wireless sensors with an acceptable accuracy [9].
- Maintenance: maintenance of wireless sensors nodes is made easier as they are smart and can assist automatic diagnosis, so any arising problems can be resolved quickly. Repairing or replacing the wireless nodes is easier because of the non-intrusive nature of WSN.

- Flexibility: the biggest advantage of wireless sensors is that they can be easily deployed and withdrawn anywhere close to the road (street furniture like: traffic signs, street lights, traffic lights etc.), which make them useable for permanent or temporary traffic measurement. Wireless sensors configurations can be customized to adapt to different purposes and environments, for example environmental sensing can be done alongside traffic surveillance.

- Wireless communication: wireless communication capability is indispensable for road-to-vehicle communication; With the emergence of smart vehicles, the origin destination matrix can now be generated without relying on surveys, as it can be done by retracing the log of the communication between the sensing nodes and the vehicle during its trip.

In summary, the new generation of wireless sensors overcome the disadvantages of the traditional sensors. They are generally small in size which make them easy to install and maintain, last longer on small batteries and they generally cost less. In addition, their wireless capabilities make the sensing network scalable and more flexible.

\section{Traffic management}

The system we propose consists of two subsystems:

\section{A. Data Acquisition Subsystem:}

The WSN technology is the main actor in this subsystem. The sensor nodes installed by all road intersections need to collect and send real-time traffic data including:

- Vehicle count.

- Vehicles speed.

- Vehicle classification.

- Occupancy.

- Environmental data.

- Vehicle re-identification, the nodes also need to be able to reidentify vehicles for origin-destination traffic map.

The communication within the subsystem must be reliable and fail-safe to maintain a degree of Quality of service. Because of its low-cost, low-power and low complexity, the standard IEEE 802.15.4, adapted by the ZigBee Alliance [10], will be used in the deployment of our Sensor Network. While other wireless technologies, like Bluetooth or WIFI, provide high data rates, they are disadvantageous due to their high-power consumption, application complexity, and cost [11].

\section{B. Control and data analysis subsystem:}

The Control Station "CS" collects and processes the data, then takes appropriate sets of predetermined actions to solve or to prevent traffic problems. The CS has three main functions:

- The CS should collect, compile then store the environmental and the relevant traffic data in a database accessible for further studying and reviewing purposes. 
- This system will use the real time traffic data to give the macroscopic or microscopic evaluation of the traffic flow in the city.

- The CS will assess traffic condition based on the collected data, then if a traffic problem is detected it will take an automated response to resolve the situation.

\section{Simulations results}

The targeted environment of our research is a smart city, which by theory will have full control over intersections lights and electric traffic signs. LED lights replace paint to make road lines and signage, which opens the possibility of changing the traffic flow by opening/blocking some roads or exchanging the number of lanes used between the two opposite directions in a specific street.

The proposed actions were simulated in VISSIM [12] software to test their feasibility and effectiveness. The subject test bed in this study is a $4 \mathrm{~km}$ stretch of national no1 going through LARACHE city from the city centre to the highway entrance, the road has 2-lanes in each direction and only 4 major intersections were simulated, the street serves all kinds of vehicles. The simulation parameters were determined after observing the traffic in the simulated area daily for the period of one week. The traffic composition was set at the default setting by the VISSIM program. The study period encompassed the evening peak hour between 5 p.m. and 6 p.m. The main inbound traffic flow was set to 1200 vehicle per hour and to 100 vehicles per hour for every other vehicle input. The inbound traffic has a $20 \%$ chance to change direction at every intersection. And every vehicle coming from a branch road to an intersection has a $90 \%$ chance to go to the inner city.

Two scenarios were simulated: the first scenario we simulated a standard situation without any change to the infrastructure or traffic signal behaviour (standard city), in the second scenario we implemented the proposed counter-actions in the same network (smart city): we changed the number of lanes for inbound direction to 3 and the opposite direction was left with one lane. We also changed the priority rules at every intersection as to prioritise the bigger flow, the traffic lights were also programmed to give more green-light time to the inbound traffic.

Each scenario was simulated several times and several traffic parameters were exported from the VISSIM evaluation lists such as average delay time, number of stops per vehicle and the amount of harmful gases emitted by the vehicles. Fig 1. to Fig. 5 show the average results of each scenario.

Fig. 1 compares the delay parameters between the two scenarios, the average delay per vehicle and average standstill time per vehicle and the latent delay (total delay time of all vehicles that could not enter the network immediately), the second scenario shows less delay time across all the parameters. Fig. 2 compares the average vehicle stops per vehicle, the vehicle in the first scenario were forced to stop in more occasions than in the second scenario. Fig 3. Compares the average traffic queue length and the maximum queue length detected in the two scenarios, our counter-measures minimized the traffic queue length.

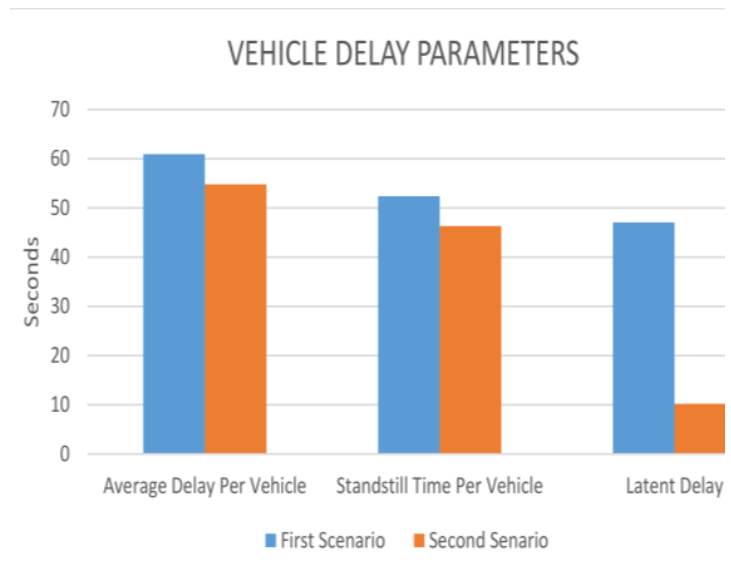

Figure 1. Vehicle delay parameters in the network.

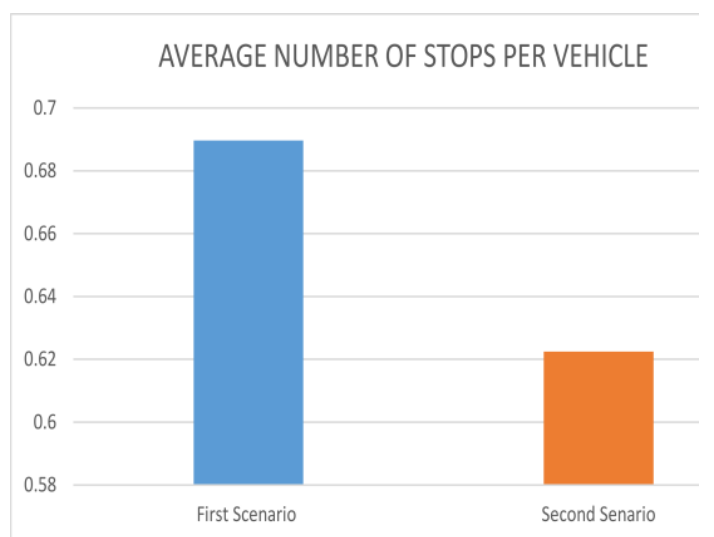

Figure 2. Average number of stops per vehicle.

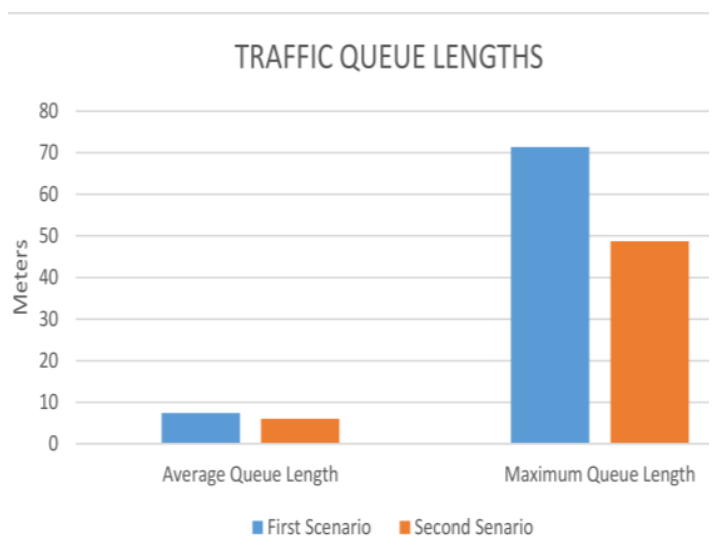

Figure 3. Average and maximum vehicle queue length measured.

The effect of increasing the number of lanes for the inbound traffic minimized the delay time and reduced traffic congestion, Also, the changing of traffic light behaviour and the priority rules for the benefits of the dense inbound traffic produced decreased the number and the duration of vehicle stops. These results show the effectivness of the proposed solutions against the traffic problems.

To complete the evaluation, we compared the environmental data extracted from the simulation to evaluate the impact of our counter-actions on the environment. Fig. 4 shows the total mass of the gases 
emitted by all vehicles in the network. The smart city had relatively less gases emissions than the standard city. The difference in speed between the two simulations is also relatively small: $36 \mathrm{~km} / \mathrm{h}$ for the first scenario, and $38 \mathrm{~km} / \mathrm{h}$ for the second. Fig. 5 compares the fuel consumed by the vehicles that travelled in the network. the second scenario

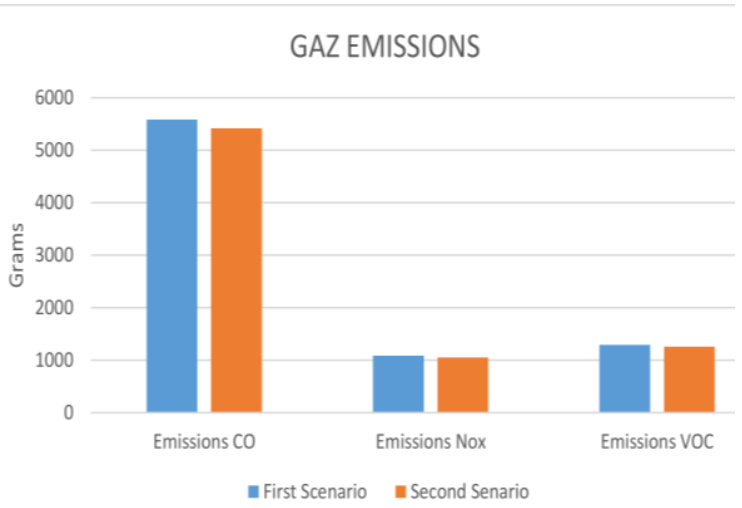

Figure 4. Vehicle emissions of CO, NOx, and Volatile Organic Compounds

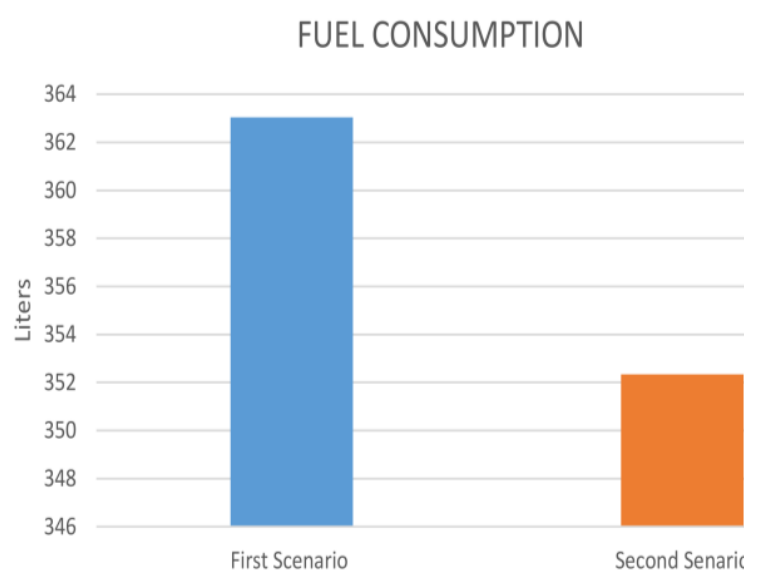

Figure 5. Vehicle emissions of CO, NOx, and Volatile Organic Compounds

had less fuel consumption which can be translated to higher fuel usage efficiency.

\section{Conclusion and future work}

In this paper we have proposed a traffic surveillance and management system based on WSN. The surveillance system is based on a network of wireless sensor nodes installed across all the intersection of the traffic network. The WSN will detect traffic parameters across the city to give an overview of the traffic status in the city. The central Unit will analyse the data and take automated counter-measures to arising traffic problems thanks to remote controlled traffic lights, road signs and lane assignment. We evaluated the counter-measures in traffic simulation software VISSIM and results showed that our plan reduced the traffic congestion, minimized gases emission and increased fuel usage efficiency.

In a future study, we intend to determine the WSN topology and routing protocols. The performance of the chosen WSN architectures will be simulated by the
CUPCARBON software to test their effectiveness and quality of service. We will re-test our smart city solutions in a bigger scale and a more complex network, we will also try to implement a customer service system that sends real-time traffic status to drivers which will help them choose faster, less congested streets, toward their destination.

\section{Acknowledgement}

We would like to express our gratitude to the PTV Group for granting a free licence to use the VISSIM software.

\section{References}

1. K. Gwilliam, World Bank. Cities on the Move : A World Bank Urban Transport Strategy Review. Washington, DC. (C) World Bank. (2002)

2. Z. BINBIN, Adaptive Traffic Light Control in Wireless Sensors Network-based Intelligent Transportation System, The Hong Kong Polytechnic University, (2010).

3. P. Varaiya and S.-Y. Cheung, Traffic Surveillance by Wireless Sensor Networks, UNIVERSITY OF CALIFORNIA, BERKELEY, final report UCB-ITSPRR-2007-4, (2007).

4. O. Karpis, Wireless sensor networks in intelligent transportation systems, Int. J. Mod. Eng. Res. IJMER, vol. 3, pp. 611-617, (2013).

5. W. Chen, L. Chen, Z. Chen, and S. Tu, Wits: A wireless sensor network for intelligent transportation system, in Computer and Computational Sciences, 2006. IMSCCS'06, vol. 2, pp. 635-641,(2006).

6. M. Franceschinis, L. Gioanola, M. Messere, R. Tomasi, M. A. Spirito, and P. Civera, Wireless sensor networks for intelligent transportation systems, in Vehicular Technology Conference, 2009. VTC Spring 2009. IEEE 69th, pp. 1-5 , (2009).

7. J. J. Fêrnández-Lozano, M. Martín-Guzmán, J. Martín-Ávila, and A. García-Cerezo, A Wireless Sensor Network for Urban Traffic Characterization and Trend Monitoring, Sensors, vol. 15, no. 10, pp. 26143-26169, (Oct. 2015).

8. P. T. Martin, Y. Feng, X. Wang, and others, Detector technology evaluation, Citeseer, (2003).

9. D. P. Mishra, G. M. Asutkar, and S. S. Dorale, An Application of Wireless Sensor Network in Intelligent Transportation System, pp. 90-91, (2013).

10. 'zigbee alliance'. Bishop Ranch, CA, http://www.zigbee.org/

11. K. Sohraby, D. Minoli, and T. F. Znati, Wireless sensor networks: technology, protocols, and applications. Hoboken, N.J: Wiley-Interscience, (2007).

12. 'PTV Vissim'. [Online]. Available: http://visiontraffic.ptvgroup.com/en-us/products/ptvvissim 